\title{
7. DIATOM BIOSTRATIGRAPHY AND PALEOCEANOGRAPHY, DEEP SEA DRILLING PROJECT LEG 681
}

\author{
Constance Sancetta, Lamont-Doherty Geological Observatory, Columbia University, Palisades, New York
}

\section{INTRODUCTION}

The primary goal of Leg 68 was the continuous recovery of undisturbed high-sedimentation-rate sections from the Caribbean and the eastern equatorial Pacific. We hoped that these sections, when correlated by highresolution paleomagnetics, would serve as stratigraphic reference sections for the various microfossil groups and would provide material to interpret paleoceanographic events, particularly those related to the closing of the Central American Seaway.

For the diatoms, the goals were partially achieved. Diatoms are essentially absent from Neogene Caribbean sediment-in fact, only one late Miocene sample contained sufficient diatoms for analysis. Interbasinal correlations and reference to tectonic events of the isthmus are thus impossible. At the Pacific site (Site $503,4^{\circ} 3^{\prime} \mathrm{N}$, $95^{\circ} 38^{\prime} \mathrm{W}$ ), diatoms occur throughout the section; however, the location of the site so close to the equator, the instability of sediment magnetism, and the inclusion of rust in the core liners resulted in an incomplete paleomagnetic record (see Kent and Spariosu, this volume).

Fortunately, this failure to provide a correlation with the magnetic record is less serious for the diatoms than for some of the other microfossil groups. Burckle (1972, 1977, 1978; Burckle and Trainer, 1979), using datum levels, has correlated the paleomagnetic record with nearly 40 diatom events of the late Neogene. The sequence and timing of these events have been tested with paleomagnetics in several equatorial Pacific and Indian Ocean sites, so that it is possible to distinguish time transgressive from synchronous events. Figure 1 shows the Leg 68 datum levels and the magnetic correlations reported by Burckle. The Pleistocene and Pliocene are subdivided for convenience of discussion-the former at the Brunhes/Matuyama boundary and the latter at the Gauss/Gilbert boundary.

\section{METHODS}

Cores from both holes at Site 503 were sampled at $50-\mathrm{cm}$ intervals (two samples per section plus core catchers), and the level for each species event was determined to the nearest sample. (At Site 502, as already indicated, only one sample contained diatoms.) For extinctions (tops) and first occurrences (bases), the datum level chosen was the last (or first) sample containing the species in reasonable numbers, rather than the first sample where the species was absent or at some intermediate level. This definition limits the range of the species to a minimum. Figure 2 shows a hypothetical case in which two species events occurred near an unrecovered interval. The first appearance of

\footnotetext{
${ }^{1}$ Prell, W. L., Gardner, J. V., et al., Init. Repts. DSDP, 68: Washington (U.S. Govt. Printing Office).
}

Species 1 could have occurred anywhere between $100 \mathrm{~cm}$ (where it is present) and $200 \mathrm{~cm}$ (where it is absent). Similarly, the extinction of Species 2 could have occurred anywhere in the same interval. The use of the first (or last) known occurrence to mark the datum level thus produces the most conservative (minimum) range for the species. This effect will be particularly marked in intervals of poor recovery.

The "true" level of a datum also depends on the consistency and abundance of occurrence of the species. For diatoms, first appearances are usually unambiguous, so that the species disappears downcore after an interval of consistent presence. Extinctions, however, often occur following a time of increasing rarity of the species, so that it is difficult to distinguish the "last occurrence" from minor reworking of individual specimens. In addition, some species are always rare, or occur sporadically, throughout their range. The only objective solution to this problem would be to count several hundred specimens in each sample and determine the abundance of the species in question. Since this approach was not feasible (because of time constraints), the datum level was chosen as the first (or last) sample in which the species was estimated to be at least $2 \%$ of the diatom assemblage (i.e., more than isolated individuals) and above (or below) which the species occurred consistently in all samples. The latter requirement obviates, to a large degree, the problem of sporadic reworking.

\section{DIATOM BIOSTRATIGRAPHY}

\section{Site $\mathbf{5 0 2}$}

Diatoms are nearly absent at Site 502 and appear only in Hole $502 \mathrm{C}$ as rare corroded fragments in the upper Miocene. Only in one sample $(502 \mathrm{C}-30-1,3 \mathrm{~cm})$ are they sufficiently frequent and well preserved to be useful. This sample contains Thalassiosira burckliana, rare Coscinodiscus endoi, and Nitzschia porteri sensu Burckle. Coscinodiscus yabei does not occur. If the stratigraphic ranges of the species here are the same as in the Pacific, this sample must lie between the lower part of Epoch 7 and the base of Epoch 8 (Fig. 1). If C. yabei is absent because of stratigraphic position (rather than because of an ecologic exclusion from the region), then the lower limit of the age can be restricted to the upper part of Epoch 8. Abbott (1980) has reported C. yabei from middle Miocene sediments of the U.S. Atlantic margin, so we know that the species occurs in the Atlantic. A tentative age for this sample is thus 7.6 to $8.2 \mathrm{~m}$.y. (the interval between the extinctions of $T$. burckliana and $C$. yabei; Fig. 1).

\section{Site 503}

Fairly well-preserved diatoms occur throughout Site 503. In general, preservation and abundance increase with depth, so that diatoms are common and well preserved in the Miocene, moderately common and well preserved in the lower part of the Pliocene, and few to rare with rather poor preservation in the upper Pliocene and Pleistocene. 


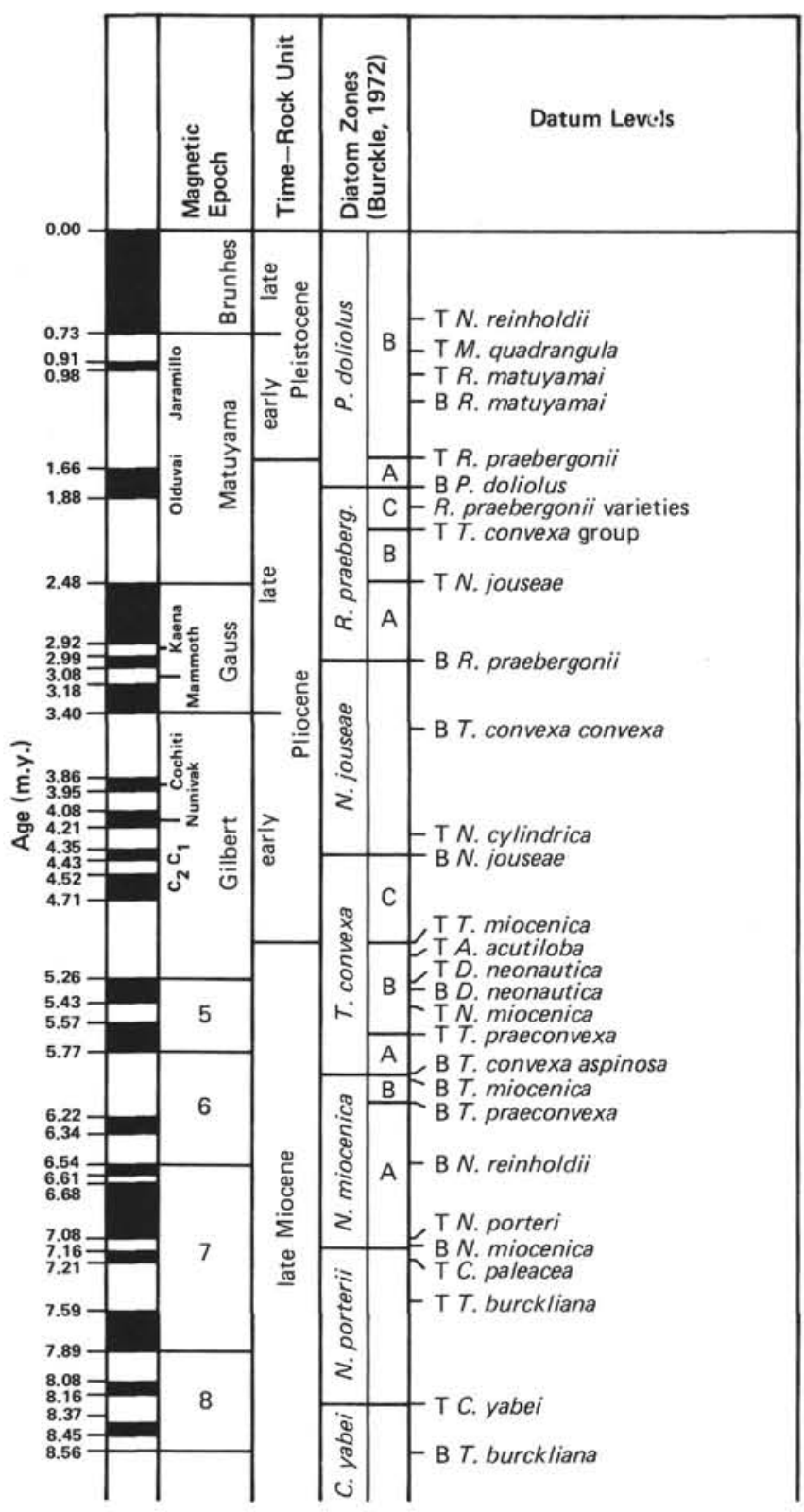

Figure 1. Diatom events used for biostratigraphy, Leg 68. (Subdivision of the Pliocene and Pleistocene is informal. $T=$ top of range [extinction]; $\mathrm{B}=$ base of range [first appearance]. Datum levels and paleomagnetic correlations from Burckle, [1972, 1977, 1978; Burckle and Trainer, 1979; Burckle et al., 1978]. Paleomagnetic dates from Mankinen and Dalrymple, 1979).

Two holes were cored continuously at Site 503 (Hole 503 was a single mudline core). Hole 503A penetrated 235 meters into the upper Miocene (av. $=62 \%$ recovery), and Hole 503B penetrated 113 meters into the lower Pliocene (av. $=85 \%$ recovery). This yields an essentially complete section for the upper 113 meters of sediment.

Tables 1 and 2 report the relative abundance of stratigraphic species in all samples. They indicate the consistency in occurrence of the species and the reliability of the datums chosen.

Figure 3 shows the recovery, species ranges, and magnetics for the two holes. In each case, the data for Hole 503B are plotted to the right of those for Hole 503A.

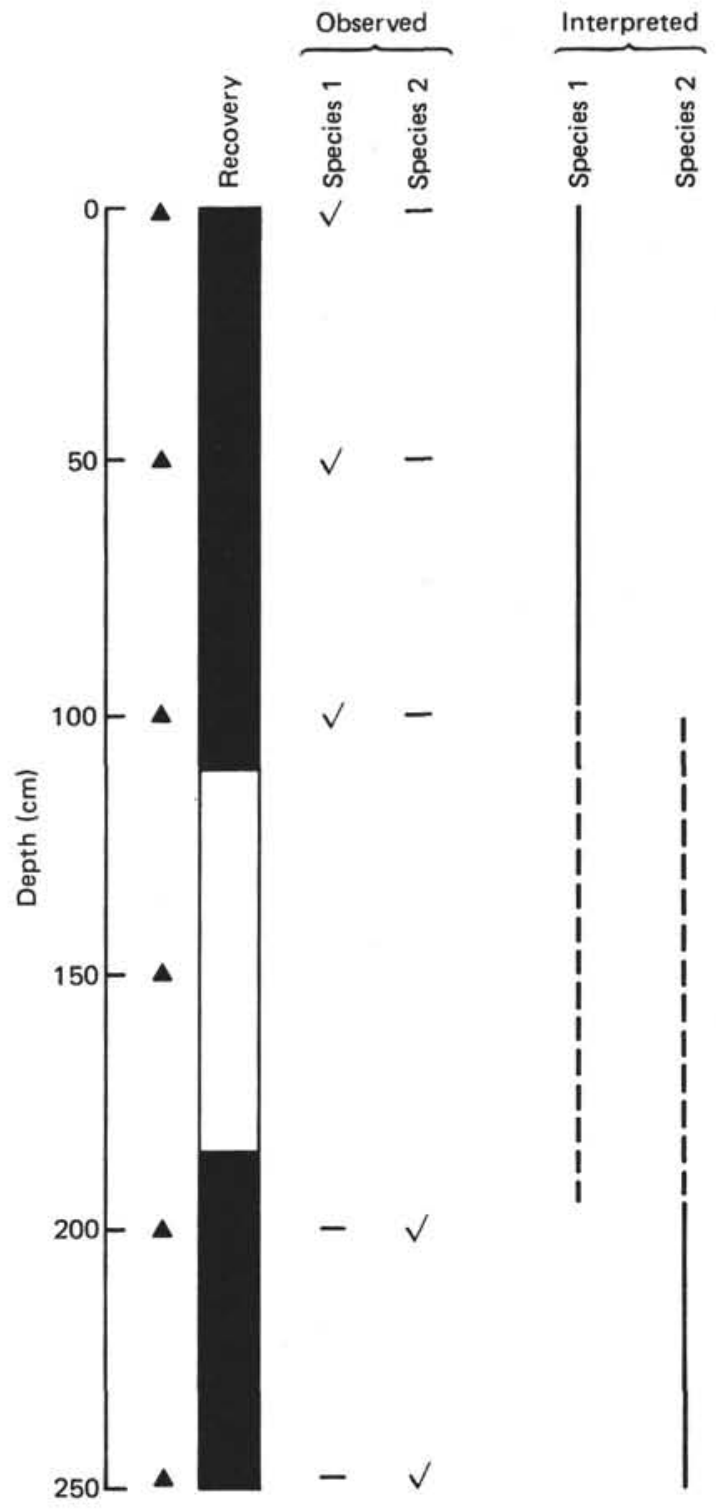

Figure 2. Hypothetical case showing effect of nonrecovery on range determination. (Triangle $=$ theoretical sampling interval; check = samples in which a species was observed; dash $=$ samples in which the species was not found; solid line $=$ the range used in this chapter, based on the sample with last [or first] presence of the species; dashed line = possible greater range if the species actually occurred in the unrecovered interval.)

Most of the events are within the same 1-meter interval in the two holes, and the worst (the base of Pseudoeunotia doliolus) is within a 3-meter interval. This suggests that there is no significant offset in depth between the two holes. The base of $P$. doliolus and the top of Nitzschia jouseae do not necessarily contradict this interpretation, as $N$. jouseae is very rare before its extinction (see Methods, this chapter), and the base of $P$. doliolus occurs in an interval of poor diatom preservation and possibly in an unrecovered interval in Hole 503B. Throughout this chapter, I assume that the depth-in-hole values of the two holes are equivalent.

The majority of the species events follow the general sequence and timing reported elsewhere by Burckle 
Table 1. Relative abundance of stratigraphic marker diatoms, Hole 503A.

\begin{tabular}{|c|c|c|c|c|c|c|c|c|c|c|c|c|}
\hline $\begin{array}{l}\text { Core/Section } \\
(\text { level in cm) }\end{array}$ & 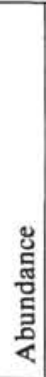 & 胥 & 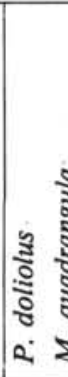 & 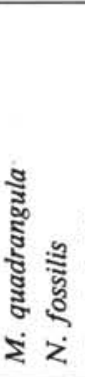 & 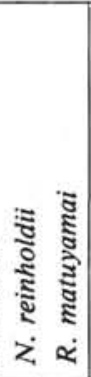 & 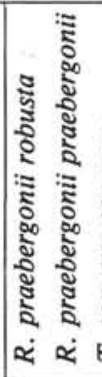 & 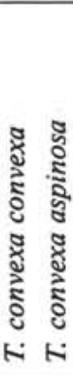 & 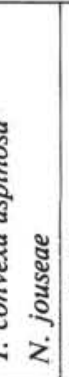 & 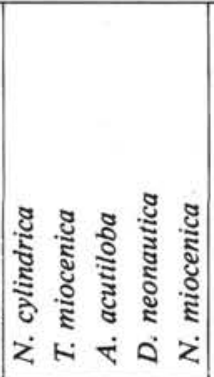 & 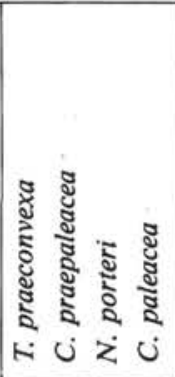 & Datum Level & 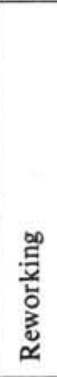 \\
\hline $\begin{array}{l}1, \mathrm{CC} \\
2, \mathrm{CC} \\
3, \mathrm{CC} \\
4-1,48 \\
4-2,48 \\
\end{array}$ & $\begin{array}{l}\mathrm{P} \\
\mathrm{R} \\
\mathrm{F} \\
\mathrm{F} \\
\mathrm{P}\end{array}$ & $\begin{array}{l}\mathrm{M} \\
\mathrm{M} \\
\mathrm{M} \\
\mathrm{G} \\
\mathrm{G}\end{array}$ & 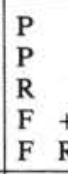 & $\begin{array}{r} \\
+\quad+ \\
\mathrm{R}+\end{array}$ & & & & & & & Top, $N$. fossilis $+M$. quadrangula & \\
\hline $\begin{array}{l}4-2,108 \\
4-3,48 \\
4, C C \\
5-1,48 \\
5-1,108\end{array}$ & $\begin{array}{l}\mathrm{F} \\
\mathrm{F} \\
\mathrm{P} \\
\mathrm{F} \\
\mathrm{R}\end{array}$ & \begin{tabular}{|c|}
$G$ \\
$G$ \\
$M$ \\
$P$ \\
$P$ \\
\end{tabular} & \begin{tabular}{|ll}
$\mathrm{R}$ & $\mathrm{F}$ \\
$\mathrm{P}$ & $\mathrm{F}$ \\
& $\mathrm{F}$ \\
$\mathrm{F}$ & $\mathrm{F}$ \\
$\mathrm{R}$ & $\mathrm{F}$ \\
\end{tabular} & $\begin{array}{l}\mathrm{F} \\
\mathrm{F} \\
\mathrm{F} \\
\mathrm{R} \\
\mathrm{R}\end{array}$ & $\begin{array}{l}\mathrm{F} \\
\mathrm{R}+ \\
+\end{array}$ & & & & & & Top, $N$. reinholdii & \\
\hline $\begin{array}{l}5-2,48 \\
5-2,108 \\
5-3,48 \\
5, \mathrm{CC} \\
6, \mathrm{CC}\end{array}$ & $\begin{array}{l}\mathrm{P} \\
\mathrm{R} \\
\mathrm{F} \\
\mathrm{F} \\
\mathrm{F}\end{array}$ & \begin{tabular}{l|}
$\mathrm{M}$ \\
$\mathrm{P}$ \\
$\mathrm{M}$ \\
$\mathrm{M}$ \\
$\mathrm{M}$
\end{tabular} & 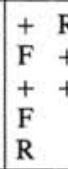 & $\begin{array}{l}\text { R } \\
+ \\
+\end{array}$ & $\mathbf{R}$ & & & & & & Base of $M$. quadrangula acme & \\
\hline $\begin{array}{l}7-1,48 \\
7-1,126 \\
7-2,48 \\
7-2,108 \\
7-3,48 \\
\end{array}$ & $\begin{array}{l}\mathrm{F} \\
\mathrm{B} \\
\mathrm{B} \\
\mathrm{B} \\
\mathrm{P} \\
\end{array}$ & G & $\begin{array}{l}\mathrm{R}+ \\
\mathrm{R}\end{array}$ & F & + & + & & & & & Top, R. praebergonii robusta & $\star$ \\
\hline $\begin{array}{l}7-3,108 \\
7, \mathrm{CC} \\
8, \mathrm{CC} \\
9-1,48 \\
9-1,108\end{array}$ & $\begin{array}{l}\mathrm{P} \\
\mathrm{P} \\
\mathrm{B} \\
\mathrm{F} \\
\mathrm{B}\end{array}$ & $\begin{array}{l}\text { G } \\
\text { G } \\
\text { G }\end{array}$ & $\begin{array}{l}\mathrm{R}+ \\
\mathrm{F} \\
\mathrm{R}\end{array}$ & 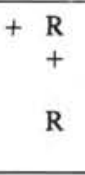 & $\begin{array}{l}\mathrm{F} \\
\mathrm{R} \\
\mathrm{R}\end{array}$ & + & & & & & . & $\begin{array}{l}\star \\
\star\end{array}$ \\
\hline $\begin{array}{l}9-2,48 \\
9-2,102 \\
9-3,48 \\
9, \mathrm{CC} \\
10, \mathrm{CC} \\
\end{array}$ & $\begin{array}{l}\mathrm{F} \\
\mathrm{P} \\
\mathrm{R} \\
\mathrm{P} \\
\mathrm{R} \\
\end{array}$ & \begin{tabular}{|l|}
$\mathrm{G}$ \\
$\mathrm{M}$ \\
$\mathrm{P}$ \\
$\mathrm{M}$ \\
$\mathrm{P}$
\end{tabular} & $\stackrel{+}{\mathrm{R}}$ & $\begin{array}{l}\mathrm{R} \\
\mathrm{R} \\
+ \\
\mathrm{R}\end{array}$ & $\begin{array}{l}\mathrm{R} \\
\mathrm{R} \\
\\
+\end{array}$ & $\begin{array}{r}+ \\
+ \\
+ \\
+ \\
+\end{array}$ & + & & & & $\begin{array}{l}\text { Top, } R . \text { praebergonii praebergonii } \\
\text { Base, } P \text {. doliolus } \\
\end{array}$ & $\begin{array}{l}\star \\
\star\end{array}$ \\
\hline $\begin{array}{l}11-2,48 \\
11-2,108 \\
11-3,48 \\
11-3,108 \\
11, \mathrm{CC} \\
\end{array}$ & $\begin{array}{l}\mathrm{F} \\
\mathrm{P} \\
\mathrm{P} \\
\mathrm{R} \\
\mathrm{P} \\
\end{array}$ & \begin{tabular}{|l|}
$\mathrm{P}$ \\
$\mathrm{M}$ \\
$\mathrm{P}$ \\
$\mathrm{P}$ \\
$\mathrm{M}$ \\
\end{tabular} & & $\begin{array}{l}\mathrm{R} \\
\mathrm{R} \\
+ \\
\mathrm{R} \\
\mathrm{F} \\
\end{array}$ & + & 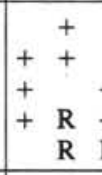 & $\begin{array}{l} \\
+ \\
+ \\
+ \\
+ \\
F\end{array}$ & & & & Top, $T$. convexa group & \\
\hline $\begin{array}{l}12, \mathrm{CC} \\
13-2,48 \\
13-2,108 \\
13-3,48 \\
13-3,108\end{array}$ & $\begin{array}{l}\mathrm{P} \\
\mathrm{P} \\
\mathrm{P} \\
\mathrm{F} \\
\mathrm{F} \\
\end{array}$ & \begin{tabular}{|l|}
$G$ \\
$G$ \\
$M$ \\
$M$ \\
$M$
\end{tabular} & & $\begin{array}{l}\mathrm{F} \\
\mathrm{F} \\
\mathrm{F} \\
\mathrm{F} \\
\mathrm{R}\end{array}$ & $\begin{array}{l}\mathrm{R} \\
\mathrm{R} \\
\mathrm{R} \\
+\end{array}$ & $\begin{array}{r}+\mathrm{R} \\
+ \\
\mathrm{F} \\
\mathrm{R}\end{array}$ & $\begin{array}{l}+ \\
\mathrm{R} \\
+ \\
+ \\
+ \\
+ \\
\mathrm{F}\end{array}$ & $\begin{array}{l}+ \\
\\
\mathrm{R} \\
\mathrm{R}\end{array}$ & & 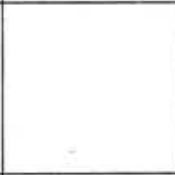 & Top, $N$. jouseae & \\
\hline $\begin{array}{l}13, \mathrm{CC} \\
14, \mathrm{CC} \\
15-2,108 \\
15-3,48 \\
15, \mathrm{CC}\end{array}$ & $\begin{array}{l}\mathrm{P} \\
\mathrm{P} \\
\mathrm{P} \\
\mathrm{P} \\
\mathrm{P} \\
\end{array}$ & \begin{tabular}{|l|}
$G$ \\
$G$ \\
$G$ \\
$G$ \\
$G$ \\
\end{tabular} & & $\begin{array}{l}\mathrm{F} \\
\mathrm{F} \\
\mathrm{R} \\
\mathrm{R} \\
\mathrm{R}\end{array}$ & $\begin{array}{l}\mathrm{R} \\
+ \\
+ \\
\mathbf{R} \\
+\end{array}$ & $\begin{array}{l}\mathbf{R} \\
\mathbf{R} \\
\mathbf{R}\end{array}$ & $\begin{array}{ll}+ & \\
\mathrm{R} & \\
\mathrm{F} & \mathrm{R} \\
\mathrm{P} & \mathrm{R} \\
\mathrm{P} & \mathrm{R} \\
\end{array}$ & $\begin{array}{l}F \\
F \\
F \\
F \\
F\end{array}$ & & & Base, $R$. praebergonii praebergonii & $\star$ \\
\hline $\begin{array}{l}16, \mathrm{CC} \\
17, \mathrm{CC} \\
18, \mathrm{CC} \\
19-1,108 \\
19-2,108\end{array}$ & $\begin{array}{l}\mathrm{P} \\
\mathrm{P} \\
\mathrm{P} \\
\mathrm{F} \\
\mathrm{P} \\
\end{array}$ & \begin{tabular}{|l|}
$G$ \\
$G$ \\
$G$ \\
$M$ \\
$M$ \\
\end{tabular} & & $\begin{array}{l}\mathrm{R} \\
+ \\
\mathbf{R} \\
\mathrm{R} \\
\end{array}$ & $\begin{array}{l}+ \\
\mathrm{R} \\
+ \\
+\end{array}$ & & $\begin{array}{ll}R & F \\
R & F \\
F & R \\
R & R \\
+ & R \\
\end{array}$ & $\begin{array}{l}F \\
F \\
R \\
F \\
F\end{array}$ & & & Base, $T$. convexa convexa & $\star$ \\
\hline $\begin{array}{l}19, \mathrm{CC} \\
20, \mathrm{CC} \\
21, \mathrm{CC} \\
22, \mathrm{CC} \\
23, \mathrm{CC}\end{array}$ & \begin{tabular}{l|}
$\mathrm{P}$ \\
$\mathrm{P}$ \\
$\mathrm{F}$ \\
$\mathrm{F}$ \\
$\mathrm{P}$
\end{tabular} & \begin{tabular}{|c|}
$G$ \\
$G$ \\
$G$ \\
$M$ \\
$G$
\end{tabular} & & $\begin{array}{l}\mathrm{F} \\
\mathbf{R} \\
\mathrm{R} \\
\mathrm{R}\end{array}$ & $\begin{array}{l}\text { R } \\
+\end{array}$ & & $\begin{array}{l}\mathrm{R} \\
\mathrm{F} \\
\mathrm{F} \\
\mathrm{R}\end{array}$ & $\begin{array}{l}F \\
R \\
R \\
R\end{array}$ & $\mathbf{R}$ & & & \\
\hline
\end{tabular}




\section{SANCETTA}

Table 1. (Continued).

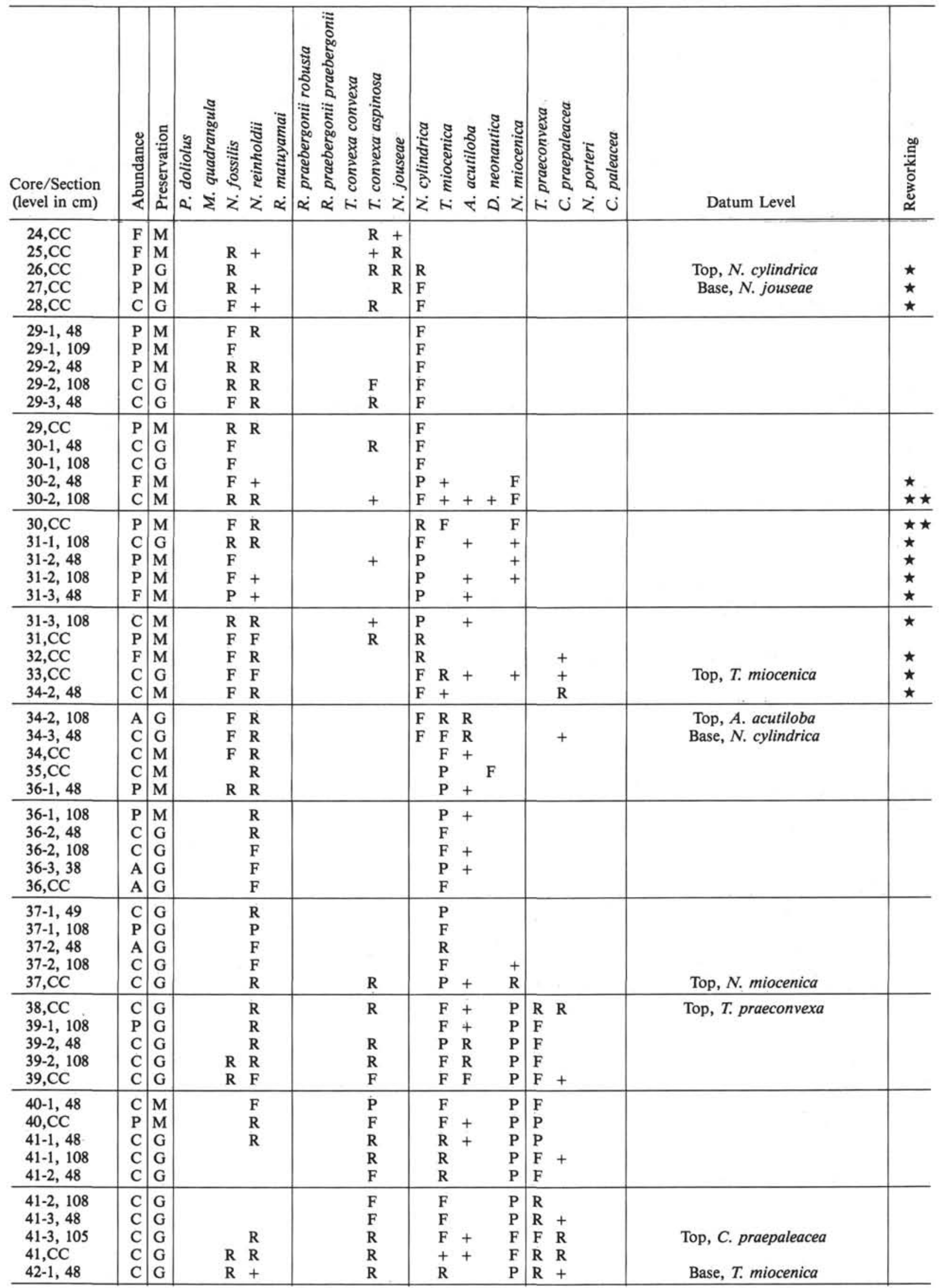


Table 1. (Continued).

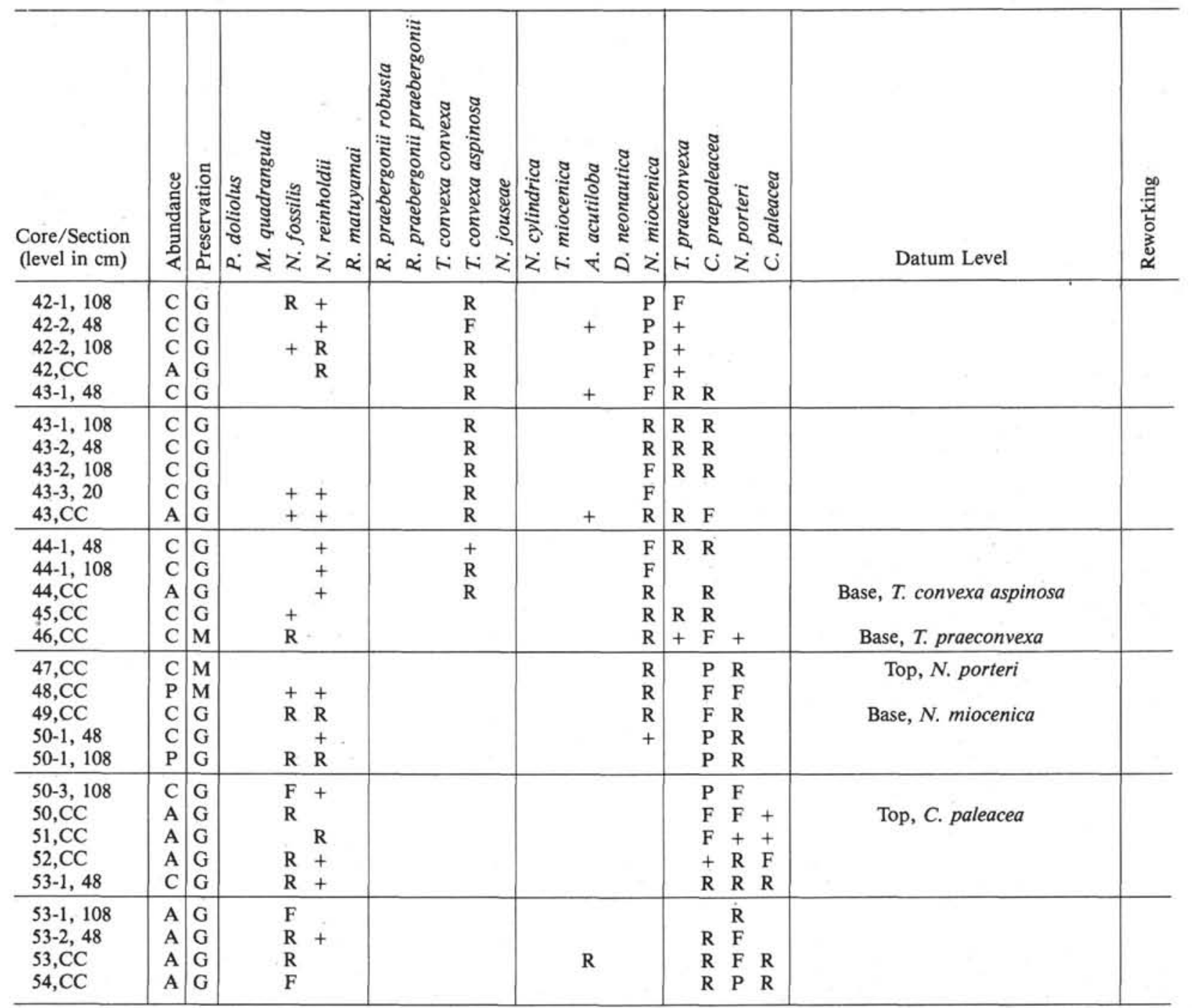

Note: Abundance and Preservation refer to diatoms as a whole in relation to other sediment components. Abundance: $\mathrm{B}=$ barren (no diatoms), $\mathrm{R}=\operatorname{rare}(<5 \%), \mathrm{F}=$ few $(5-15 \%), \mathrm{P}=$ present $(15-40 \%), \mathrm{C}=$ common $(40-60 \%), \mathrm{A}=$ abundant $(>60 \%)$. Preservation: $\mathrm{P}=$ poor (broken, corroded, only resistant species); $\mathrm{M}=$ moderate, $\mathrm{G}=$ good (fine structures preserved; weakly silicified forms present). Abundance of individual species is relative to diatom assemblage as a whole. $+=$ isolated specimens only; other values as for total abundance. $\star=$ samples with significant reworking (one = minor; two stars = serious reworking). The sample chosen for each datum is indicated.

(1977, 1978; Burckle and Trainer, 1979), although his correlations with the magnetics cannot be verified in the lower portion. The most distinctive differences are those for the Nitzschiaceae (Nitzschia and Pseudoeunotia). Only $N$. porteri, $N$. miocenica, and $N$. jouseae appear to conform to the sequence established by Burckle.

Burckle's only use of $N$. cylindrica has been the time of its evolutionary transition to $N$. jouseae (4.4-4.3 m.y.; Fig. 1). In later work (Burckle, 1978), he has rated this datum at a low level of reliability, since $N$. cylindrica has a rather sporadic occurrence, geographically and temporally. At Site 503, however, $N$. cylindrica consistently occurs in moderately low numbers from the level of first appearance in the uppermost Miocene to its disappearance in the lower Pliocene (Table 1, Fig. 3). It may thus be useful as a secondary marker in this more eastern part of the equatorial Pacific.
Burckle and Trainer (1979) used the mid-Olduvai for the first appearance of $P$. doliolus. At Site 503, I found rare but distinctive specimens as deep as 38 meters (Table 2), which is just below the Olduvai (Kent and Spariosu, this volume). This may reflect a true diachroneity in the appearance of the species or may be caused by a different conception of the morphologic limits of the species.

Burckle has not used the extinction of $N$. fossilis in his stratigraphy, but the level of this event at Site 50312 meters, near the Brunhes/Matuyama boundarycorresponds to that reported by Kazarina (1975) in the eastern equatorial Indian Ocean. This event may be a reliable boundary marker in equatorial regions.

Finally, the range of $N$. reinholdii at Site 503 differs from that reported by Burckle. He found the extinction of $N$. reinholdii in the lowermost Brunhes (Burckle, 
Table 2. Relative abundance of stratigraphic marker diatoms, Hole 503B.

\begin{tabular}{|c|c|c|c|c|c|c|c|c|c|c|c|}
\hline $\begin{array}{l}\text { Core/Section } \\
\text { (level in cm) }\end{array}$ & 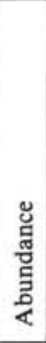 & 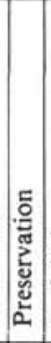 & 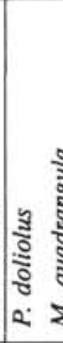 & 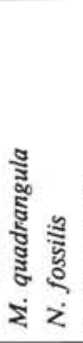 & 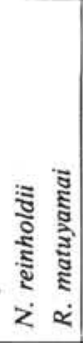 & 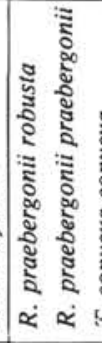 & 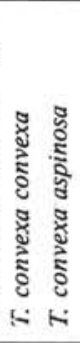 & 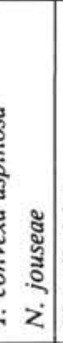 & 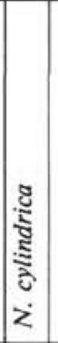 & Datum Level & 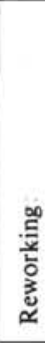 \\
\hline $\begin{array}{l}1, \mathrm{CC} \\
2, \mathrm{CC} \\
3-2,40 \\
3-2,100 \\
3-3,40\end{array}$ & $\begin{array}{l}\mathrm{P} \\
\mathrm{R} \\
\mathrm{R} \\
\mathrm{R} \\
\mathrm{R} \\
\end{array}$ & $\begin{array}{l}\mathrm{M} \\
\mathrm{P} \\
\mathrm{P} \\
\mathrm{P} \\
\mathrm{P}\end{array}$ & $\begin{array}{l}\mathrm{R} \\
\mathrm{R} \\
\mathrm{R} \\
\mathrm{R} \\
\mathrm{R} \\
\end{array}$ & $\stackrel{+}{\mathrm{R}}+$ & & & & & & $\begin{array}{c}\text { Top, } M . \text { quadrangula } \\
\text { Top, } N \text {. fossilis and } N \text {. reinholdii }\end{array}$ & \\
\hline $\begin{array}{l}3-3,100 \\
3, C C \\
4-1,40 \\
4-1,100 \\
4-2,40\end{array}$ & $\begin{array}{l}\mathrm{R} \\
\mathrm{R} \\
\mathrm{R} \\
\mathrm{R} \\
\mathrm{R}\end{array}$ & \begin{tabular}{|l|}
$\mathrm{P}$ \\
$\mathrm{M}$ \\
$\mathrm{P}$ \\
$\mathrm{M}$ \\
$\mathrm{P}$
\end{tabular} & $\begin{array}{ll}\mathrm{F} & - \\
\mathrm{R} & \mathrm{F} \\
\mathrm{F} & \mathrm{I} \\
\mathrm{F} & \mathrm{1} \\
& \mathrm{F}\end{array}$ & $\begin{array}{l}+ \\
\mathrm{R}+ \\
\mathrm{F}+ \\
\mathrm{F} \mathrm{R} \\
\mathrm{R}+\end{array}$ & $\begin{array}{l}+ \\
+ \\
+ \\
+ \\
+\end{array}$ & & & & & & $\star$ \\
\hline $\begin{array}{l}4-2,100 \\
4-3,40 \\
4-3,100 \\
4, C C \\
5-2,40 \\
\end{array}$ & $\begin{array}{l}\mathrm{F} \\
\mathrm{R} \\
\mathrm{R} \\
\mathrm{F} \\
\mathrm{F} \\
\end{array}$ & \begin{tabular}{|l|}
$\mathrm{M}$ \\
$\mathrm{P}$ \\
$\mathrm{P}$ \\
$\mathrm{P}$ \\
$\mathrm{M}$
\end{tabular} & $\begin{array}{lll} & & \\
R & \\
& 1 \\
R & 1 \\
F & F\end{array}$ & $\begin{array}{ll}R & \\
+ & R \\
F & R \\
F & R \\
R & R \\
\end{array}$ & $\begin{array}{l}+ \\
R \\
R \\
R \\
+\end{array}$ & & & & & 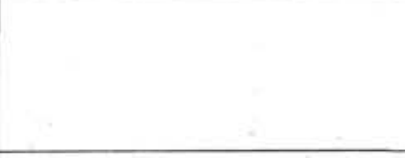 & \\
\hline $\begin{array}{l}5-2,100 \\
5-3,40 \\
5-3,100 \\
5, C C \\
6-1,100 \\
\end{array}$ & $\begin{array}{l}\mathrm{R} \\
\mathrm{F} \\
\mathrm{R} \\
\mathrm{F} \\
\mathrm{R} \\
\end{array}$ & \begin{tabular}{l|}
$\mathrm{P}$ \\
$\mathrm{M}$ \\
$\mathrm{P}$ \\
$\mathrm{M}$ \\
$\mathrm{M}$
\end{tabular} & $\begin{array}{l}\mathrm{R} \\
\mathrm{R} \\
\mathrm{R} \\
\mathrm{F} \\
\mathrm{R} \\
\end{array}$ & $\begin{array}{l}R+ \\
+R \\
+R \\
+R \\
+ \\
\end{array}$ & $\begin{array}{l}\mathrm{R} \\
+ \\
\mathrm{R}\end{array}$ & + & & & & Base, $M$. quadrangula acme & \\
\hline $\begin{array}{l}6, \mathrm{CC} \\
7-1,110 \\
7-2,40 \\
7-2,110 \\
7, \mathrm{CC}\end{array}$ & $\begin{array}{l}\mathrm{R} \\
\mathrm{R} \\
\mathrm{R} \\
\mathrm{R} \\
\mathrm{R} \\
\end{array}$ & \begin{tabular}{|l|}
$\mathrm{P}$ \\
$\mathrm{P}$ \\
$\mathrm{P}$ \\
$\mathrm{P}$ \\
$\mathrm{P}$
\end{tabular} & \begin{tabular}{|l}
$\mathrm{R}$ \\
$\mathrm{F}$ \\
$\mathrm{R}$ \\
$\mathrm{R}$ \\
\end{tabular} & $\begin{array}{l}\mathrm{R} \\
\mathrm{F} \\
+ \\
\end{array}$ & $\begin{array}{l}\mathrm{R} \\
\mathrm{F} \\
\mathrm{R} \\
\mathrm{R} \\
\end{array}$ & $\begin{array}{l}+ \\
+ \\
+\end{array}$ & $\begin{array}{l}+ \\
+\end{array}$ & & & Top, $R$. praebergonii robusta & $\star \star$ \\
\hline $\begin{array}{l}8, \mathrm{CC} \\
9, \mathrm{CC} \\
10-1,40 \\
10-1,110 \\
10-2,40\end{array}$ & $\begin{array}{l}\mathrm{R} \\
\mathrm{B} \\
\mathrm{R} \\
\mathrm{R} \\
\mathrm{R} \\
\end{array}$ & \begin{tabular}{|l|}
$\mathrm{P}$ \\
$\mathrm{P}$ \\
$\mathrm{P}$ \\
$\mathrm{P}$
\end{tabular} & $\begin{array}{l}\mathrm{R} \\
\mathrm{R}\end{array}$ & $\begin{array}{l}+ \\
\text { R } \\
\text { R }\end{array}$ & $\begin{array}{l}+ \\
+ \\
+ \\
+ \\
+\end{array}$ & $\begin{array}{l}+ \\
+\end{array}$ & & & & Base, P. doliolus & $\star$ \\
\hline $\begin{array}{l}10-3,94 \\
10, C C \\
11-1,110 \\
11-2,110 \\
11-3,40\end{array}$ & $\begin{array}{l}\mathrm{R} \\
\mathrm{F} \\
\mathrm{F} \\
\mathrm{F} \\
\mathrm{F} \\
\end{array}$ & \begin{tabular}{|l|} 
\\
$M$ \\
$M$ \\
$P$ \\
$M$ \\
\end{tabular} & + & $\begin{array}{l}\mathrm{F} \\
\mathrm{F} \\
\mathrm{F} \\
\mathrm{F} \\
\end{array}$ & $\begin{array}{l}+ \\
\mathrm{R} \\
+ \\
+\end{array}$ & $\begin{array}{ll}R & \\
F & R \\
R & R \\
R & R\end{array}$ & & & & & \\
\hline $\begin{array}{l}11-3,110 \\
11, \mathrm{CC} \\
12, \mathrm{CC} \\
13-3,40 \\
13, \mathrm{CC}\end{array}$ & $\begin{array}{l}\mathrm{F} \\
\mathrm{F} \\
\mathrm{F} \\
\mathrm{F} \\
\mathrm{F} \\
\end{array}$ & $\begin{array}{l}\mathrm{M} \\
\mathrm{M} \\
\mathrm{M} \\
\mathrm{M} \\
\mathrm{M}\end{array}$ & & $\begin{array}{l}\mathrm{F} \\
\mathrm{F} \\
\mathrm{F}\end{array}$ & $\begin{array}{l}+ \\
+ \\
+\end{array}$ & $\begin{array}{ll}\mathrm{R} & \mathrm{F} \\
\mathrm{F} \\
\mathrm{F} \\
\mathrm{R} \\
\end{array}$ & $\begin{array}{l}R \\
R \\
R \quad R \\
+\quad R \\
+\quad R \\
\end{array}$ & $\begin{array}{r}2 \\
+ \\
+ \\
+ \\
+\end{array}$ & & $\begin{array}{c}\text { Top, } T \text {. convexa group } \\
\text { Base, } R \text {. praebergonii robusta }\end{array}$ & \\
\hline $\begin{array}{l}14-1,110 \\
14, C C \\
15-1,40 \\
15-1,110 \\
15-2,40\end{array}$ & $\begin{array}{l}\mathrm{F} \\
\mathrm{P} \\
\mathrm{F} \\
\mathrm{F} \\
\mathrm{P} \\
\end{array}$ & \begin{tabular}{l|}
$M$ \\
$M$ \\
$M$ \\
$P$ \\
$M$
\end{tabular} & & $\begin{array}{l}F \\
F \\
R \\
R \\
F \\
\end{array}$ & $\begin{array}{l}+ \\
+ \\
+ \\
\mathrm{R}\end{array}$ & $\begin{array}{l}\mathrm{F} \\
\mathrm{R} \\
\mathrm{R} \\
+ \\
\end{array}$ & $\begin{array}{ll}+ & + \\
+ & R \\
F & R \\
R & R \\
+ & + \\
\end{array}$ & $\begin{array}{r}F \\
R \\
F \\
F \\
F\end{array}$ & & $\begin{array}{c}\text { Top, } N . \text { jouseae } \\
\text { Base, } R . \text { praebergonii praebergonii }\end{array}$ & \\
\hline $\begin{array}{l}15-2,110 \\
15, \mathrm{CC} \\
16, \mathrm{CC} \\
17, \mathrm{CC} \\
18, \mathrm{CC}\end{array}$ & $\begin{array}{l}\mathrm{P} \\
\mathrm{C} \\
\mathrm{F} \\
\mathrm{F} \\
\mathrm{F} \\
\end{array}$ & $\begin{array}{l}\mathrm{M} \\
\mathrm{M} \\
\mathrm{M} \\
\mathrm{M} \\
\mathrm{M}\end{array}$ & & $\begin{array}{l}\mathrm{R} \\
\mathrm{R} \\
\mathrm{F} \\
\mathrm{R} \\
\mathrm{R}\end{array}$ & $\begin{array}{l}\mathrm{R} \\
\mathrm{R} \\
\mathrm{F} \\
+ \\
+\end{array}$ & & $\begin{array}{ll}\mathrm{F} & \mathrm{F} \\
\mathrm{F} & \mathrm{R} \\
\mathrm{R} & \mathrm{R} \\
\mathrm{F} & \mathrm{F} \\
\mathrm{R} & \mathrm{R} \\
\end{array}$ & $\begin{array}{l}F \\
R \\
F \\
R \\
R\end{array}$ & & Base, $T$. convexa convexa & \\
\hline $\begin{array}{l}19-1,110 \\
19-2,40 \\
19, \mathrm{CC} \\
20, \mathrm{CC} \\
21, \mathrm{CC}\end{array}$ & $\begin{array}{l}\mathrm{C} \\
\mathrm{P} \\
\mathrm{P} \\
\mathrm{P} \\
\mathrm{P} \\
\end{array}$ & \begin{tabular}{l|}
$G$ \\
$M$ \\
$G$ \\
$G$ \\
$G$ \\
\end{tabular} & & $\begin{array}{l}\mathrm{R} \\
\mathrm{F} \\
\mathrm{F} \\
\mathrm{F}\end{array}$ & $\begin{array}{l}\mathrm{R} \\
\mathrm{F} \\
\mathrm{R} \\
\end{array}$ & & $\begin{array}{r}+R \\
R \\
F \\
\end{array}$ & $\begin{array}{l}\mathrm{R} \\
\mathrm{F} \\
\mathrm{R} \\
\mathrm{F} \\
\mathrm{R}\end{array}$ & & & \\
\hline $\begin{array}{l}22, \mathrm{CC} \\
23, \mathrm{CC} \\
24, \mathrm{CC} \\
25, \mathrm{CC} \\
26, \mathrm{CC}\end{array}$ & $\begin{array}{l}\mathrm{P} \\
\mathrm{P} \\
\mathrm{P} \\
\mathrm{P} \\
\mathrm{P}\end{array}$ & \begin{tabular}{l|}
$G$ \\
$G$ \\
$G$ \\
$G$ \\
$G$
\end{tabular} & & $\begin{array}{l}\mathrm{P} \\
\mathrm{P} \\
\mathrm{P} \\
\mathrm{P} \\
\mathrm{F}\end{array}$ & R & & $\begin{array}{l}R \\
R \\
P \\
R\end{array}$ & $\begin{array}{l}F \\
R \\
F \\
P \\
F\end{array}$ & $\begin{array}{l}\mathrm{F} \\
\mathrm{P}\end{array}$ & Top, N. cylindrica & \\
\hline
\end{tabular}

Note: For sýmbols, see note, Table 1. 


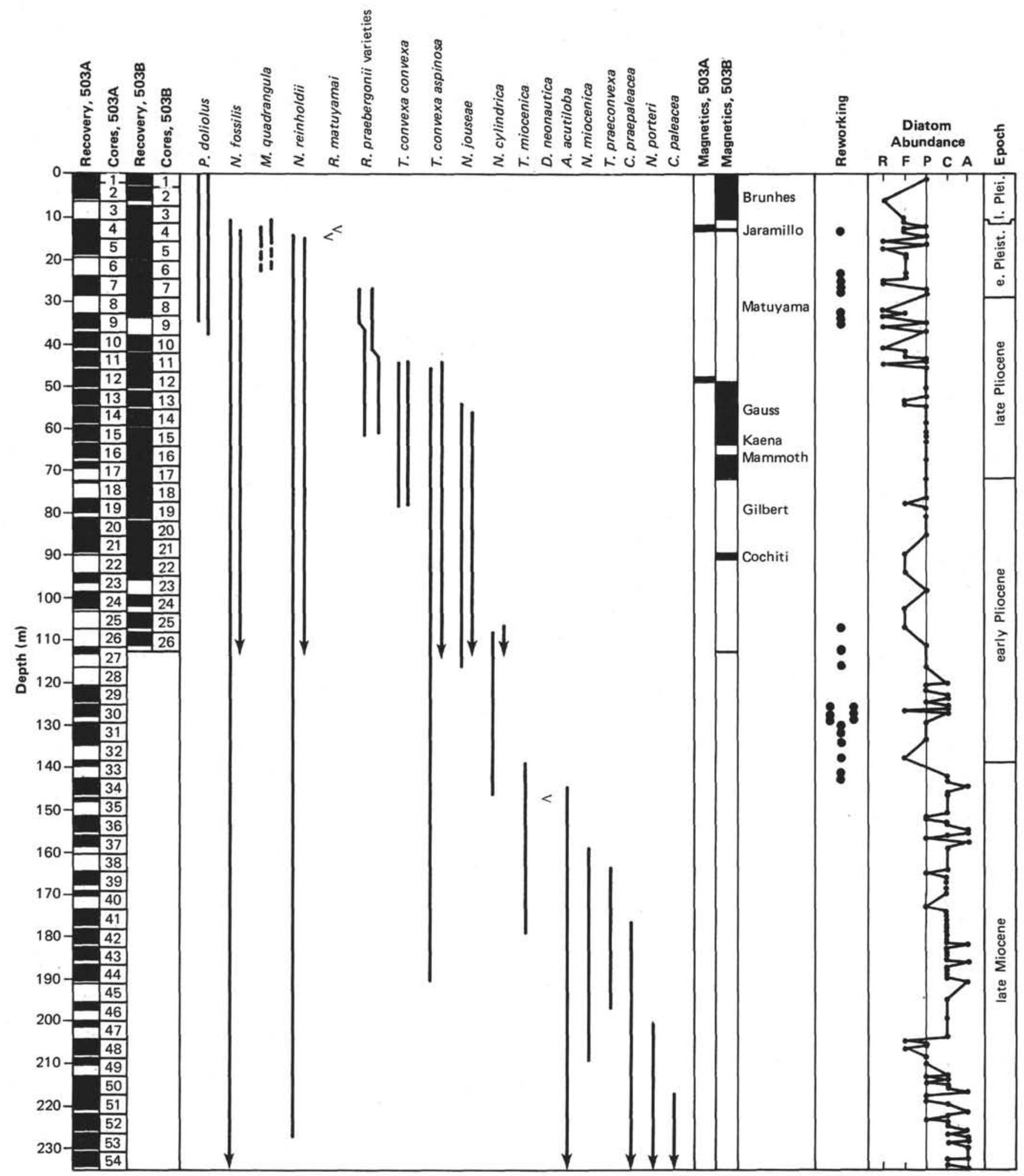

Figure 3. Diatom abundance and ranges of stratigraphic species, Site 503. (For core recovery, species ranges, and magnetics, values for Hole 503B are to the right of those for 503A. For diatom abundance, see caption Table 1. Arrows at base of range line indicate possible continuation below the last core; caret $=$ occurrence in one sample only.) 
1977) in the central equatorial Pacific and in higher latitudes (as has Koizumi, 1975), whereas at Site 503 it disappears just below the Jaramillo (Table 1, Fig. 3). The extinction is also earlier in the easternmost equatorial Pacific at Sites 504 and 505 (Sancetta, in press). According to Burckle, the first appearance of this species is in lowermost Epoch 6, between the base of Thalassiosira praeconvexa and the top of $N$. porteri (Fig. 1), whereas at Site $503 \mathrm{~N}$. reinholdii occurs, although rarely and sporadically, below the top of Cussia paleacea (Table 1, Fig. 3), which would place it in the middle of Epoch 7 (by extrapolation from Fig. 1).

It may be that the Nitzschiaceae are more sensitive to environmental effects that some of the other groupsthe very fact that they do show significant evolution during the late Neogene (at least nine events in 7 m.y.) indicates that they were evolving rather rapidly. The sporadic occurrences of $N$. reinholdii, for instance (Table 1), suggest that the species could not maintain a constant population in the eastern equatorial Pacific but experienced occasional migrations and exclusions. Stratigraphic occurrence in the region, therefore, may depend partly on ecological restrictions.

Mesocena quadrangula (a silicoflagellate) appears to have a reliable extinction just above the Jaramillo, as reported previously by Burckle (1977); however, individuals extend downward throughout the upper Neogene, so that a first appearance has not been identified. Mesocena quadrangula does show a sharp increase in abundance (acme) shortly before its extinction (Bukry and Foster, 1973), and it is the base of this acme that Burckle (1977) reported as a first appearance.

Rhizosolenia matuyamai was originally reported in the North Pacific $\left(33-40^{\circ} \mathrm{N}\right.$; Burckle et al., 1978), where it has a very short range just below and into the Jaramillo. The occurrence at Site 503 is the first reported for the equatorial region. It is present only in one sample in each hole (Tables 1,2 ), so no range can be given. It is, however, significant that a short-ranging, cool-water species did penetrate to the equatorial Pacific.

The shift from Rhizosolenia praebergonii praebergonii to $R$. praebergonii robusta occurs somewhere in the lower Matuyama at Site 503, but quantitative work must determine the rate at which it occurs. It may occupy a minor but significant time interval, rather than occurring at a single level.

Only one sample, in an interval of poor recovery, contains Dictyocha neonautica (Table 1). No range can be given for this silicoflagellate. Burckle (personal communication) found that it has a short range just below the Mio/Pliocene boundary, which agrees with the appearance at Site 503 .

The first appearances of Thalassiosira miocenica and $T$. convexa aspinosa appear to be reversed in order at Site 503 when compared to Burckle's (1978) findings (Table 1, Figs. 1, 3). This may be explained by a different conception of the species, because the two are very similar in the early part of the their ranges; however, Site 503, with much faster sedimentation rates than the cores used by Burckle, may in fact provide a more complete representation of events.

\section{PALEOCEANOGRAPHY}

Throughout the late Miocene, productivity of diatoms appears to have been high in the equatorial $\mathrm{Pa}$ cific. The diatoms are common and very well preserved-in low-carbonate intervals, the sediment approaches a diatom ooze. The assemblage is characterized by an overwhelming predominance of the species of Thalassionema and Thalassiothrix (hereafter referred to as the Thalassionema group). These pennate species are characteristic of the plankton in regions of deepwater productivity and upwelling (Hasle, 1960; Hasle and de Mendiola, 1967; Abbott and Cielsieski, personal communication) and also dominate modern sediment below the Kuroshio Current (Sancetta, 1979), the PeruChile Current, and the Antarctic Convergence-(Burckle, personal communication). Bukry (1973) and Bukry and Foster (1973) reported a predominance of the Thalassionema group in other latest Miocene equatorial sites (Sites 166, 158, 157). The abundance of this group in the upper Miocene at Site 503, coupled with the overall abundance of diatoms, implies a period of high productivity sustained by significant upwelling. Leinen (1979) showed that opal productivity in the central equatorial Pacific reached a peak at this time. Significant evolution occurred among the diatoms: Three species appeared and became extinct within a 2-m.y. interval, and several other extinctions or first appearances also occurred. The rapid turnover of the three species (Nitzschia miocenica, Thalassiosira praeconvexa and T. miocenica) suggests a good deal of variability in the environment, and the cluster of extinctions near the Mio/Pliocene boundary implies some deterioration, or even a minor crisis, for the siliceous phytoplankton.

Just above the Mio/Pliocene boundary there is an interval of major reworking (Cores 503-30-503-33 at 125$142.6 \mathrm{~m}$ ). Within this same interval the overall abundance of diatoms, and particularly of the Thalassionema group, begins to decrease. This seems to imply a drop in opal productivity and significant activity of the bottom water (but not an increase in corrosivity, because the carbonate curve shows no significant decrease; Gardner, this volume). Brewster (1980) and Leinen (1979) note that in the earliest Pliocene opal accumulation shifted from the equatorial Pacific to the Antarctic Convergence, probably as an effect of strengthening of the polar front with consequent trapping of opal in the Antarctic circumpolar region.

Through most of the Pliocene, diatoms are only moderately common and well preserved. Relatively few evolutionary events are recorded in the lower Pliocene. Conditions for the siliceous phytoplankton apparently were fairly stable. This is particularly interesting, because the initiation of northern hemisphere glaciation and the uplift of the Isthmus of Panama probably occurred during this time (Keigwin, 1978; Shackleton and Opdyke, 1977; Keigwin, this volume). At Sites 504 and 505 , in the easternmost equatorial Pacific, abundance and preservation of diatoms increased markedly, and preservation of calcium carbonate worsened about $3 \mathrm{Ma}$ (Sancetta, in press). It may be that Site 503, about 1000 
$\mathrm{km}$ to the west, is too far from the Isthmus and the region of major upwelling to show a similar effect. The primary currents of the equatorial system - the North Equatorial Current and the Equatorial Countercurrent-may have been sufficiently stable, as they are today, that local changes to the east were not drastic enough to affect the diatoms at Site 503.

In the middle of the late Pliocene, diatom abundance and preservation declined at Site 503 (Fig. 3) and remained low throughout the rest of the record. The late Pliocene and Pleistocene were probably unfavorable for diatom production. There is an interval of minor but significant reworking (of Miocene species) at the Plio/ Pleistocene boundary. Diatoms and calcareous nannofossils are poorly preserved in this interval, and the carbonate curve shows a definite minimum (Gardner, this volume). Hays et al. (1969) noted this carbonate low but made no particular environmental interpretations. The effect is even more marked at Sites 504 and 505 to the east (Sancetta, in press). The combination of low carbonate, reworking, and silica dissolution suggests a period of decreased surface productivity and increased erosion by bottom waters. The Miocene species $(N$. miocenica, Asterolampra acutiloba, Cussia praepaleacea) may have been transported from an outcrop on a nearby oceanic high.

Diatoms are rare and not well preserved through the Pleistocene. It is thus difficult to interpret any events during this period. It may be significant that two diatoms and a silicoflagellate show extinctions near the Jaramillo within a 0.5 -m.y. interval. It is in this same interval that the cool-water (?)R. matuyamai occurs (Fig. 3 ). Whereas this may be an intrusion of some other water type, it may also be an artifact of the overall rarity of diatoms (see discussion of the Nitzschia species in the biostratigraphic section, Site 503 chapter, this volume).

\section{ACKNOWLEDGMENTS}

The author thanks Professors Hans Schrader and Lloyd H. Burckle for carefully reviewing the manuscript.

\section{REFERENCES}

Abbott, W. H., 1980. Diatoms and stratigraphically significant silicoflagellates from the Atlantic Margin Coring Project and other Atlantic margin sites. Micropaleontology, 26:49-80.

Brewster, N. A., 1980. Cenozoic biogenic silica sedimentation in the Antarctic Ocean. Geol. Soc. Am. Bull., 91:337-347.
Bukry, D., 1973. Phytoplankton stratigraphy, Central Pacific Ocean, Deep Sea Drilling Project, Leg 17. In Winterer, E. L., Ewing, J. I., et al., Init. Repts. DSDP, 17: Washington (U.S. Govt. Printing Office), 871-890.

Bukry, D., and Foster, J. H., 1973. Silicoflagellate and diatom stratigraphy, Leg 16, Deep Sea Drilling Project. In van Andel, Tj. H., Heath, G. R., et al., Init. Repts. DSDP, 16: Washington (U.S. Govt. Printing Office), 815-872.

Burckle, L. H., 1972. Late Cenozoic planktonic diatom zones from the eastern equatorial Pacific. Nova Hedwig. Beih., 39:217-246. 1977. Pliocene and Pleistocene diatom datum levels from the equatorial Pacific. Quat. Res., 7:330-340. 1978. Early Miocene to Pliocene diatom datum levels for the equatorial Pacific. Spec. Pub. Indones. Geol. Res. Dev. Cent., $1: 25-44$.

Burckle, L. H., Hammond, S. R., and Seyb, S. M., 1978. A stratigraphically important new diatom from the Pleistocene of the North Pacific. Pac. Sci., 32:209-214.

Burckle, L. H., and Trainer, J., 1979. Middle and Late Pliocene diatom datum levels from the central Pacific. Micropaleontology, $25: 281-293$.

Hasle, G. R., 1960. Phytoplankton and ciliate species from the tropical Pacific. Skr. Nor. Vidensk. Akad. Math. Naturvis. Kl., (Oslo), $2: 1-50$.

Hasle, G. R., and de Mendiola, B. R. E., 1967. The fine structure of some Thalassionema and Thalassiothrix species. Phycology, 6: 107-125.

Hays, J. D., Saito, T., Opdyke, N. D., and Burckle, L. H., 1969. Pliocene-Pleistocene sediments of the equatorial Pacific: Their paleomagnetic, biostratigraphic, and climatic record. Geol. Soc. Am. Bull., 80:1481-1514.

Kazarina, G. K., 1975. Diatom zones in the sediments of the eastern tropical region of the Indian Ocean. Okeanology, 15:1073-1078.

Keigwin, L. D., 1978. Pliocene closing of the Isthmus of Panama, based on biostratigraphic evidence from nearby Pacific Ocean and Caribbean Sea cores. Geology, 6:630-634.

Koizumi, I., 1975. Diatom events in Late Cenozoic deep-sea sequences in the North Pacific. Chishitsugaku Zasshi, 81:567-578.

Leinen, M.; 1979. Biogenic silica accumulation in the central equatorial Pacific and its implications for Cenozoic paleoceanography: Summary. Geol. Soc. Am. Bull., 90:801-803.

Mankinen, E. A., and Dalrymple, G. B., 1979. Revised geomagnetic polarity time scale for the interval 0-5 m.y. BP. J. Geophys. Res., $84: 615-626$

Sancetta, C. A., 1979. Oceanography of the North Pacific during the last 18,000 years: Evidence from fossil diatoms. Mar. Micropaleontol., 4:103-123.

, in press. Biostratigraphic and paleoceanographic events in the eastern equatorial Pacific: Results of DSDP Leg 69. In Langseth, M. G., Cann, J. R., et al., Init. Repts. DSDP, 69: Washington (U.S. Govt. Printing Office).

Shackleton, N. J., and Opdyke, N. D., 1977. Inception of northern hemisphere glaciation: Oxygen isotope and paleomagnetic evidence. Nature, 270:216-218. 